\title{
Acute epiglottitis in children and adults in Sweden 1981-3
}

\author{
B Trollfors, O Nylén, K Strangert
}

\begin{abstract}
In a retrospective study of the incidence of acute epiglottitis in Sweden, 485 children and 356 adults fulfilled the following criteria: (a) red and swollen epiglottis visualised at laryngoscopy; (b) stridor or difficulties in swallowing own saliva or water; and (c) temperature $\geqslant 38^{\circ} \mathrm{C}$. The age specific incidence in children (0-14 years) was 10 and in adults ( $\geqslant 15$ years) $1 \cdot 8 / 100000 /$ year. These incidence rates were higher than the incidence of Haemophilus influenzae meningitis in the same population. Blood cultures were obtained from 290 children (60\%) and 185 adults (52\%). $H$ influenzae was isolated from 267 blood cultures (92\%) from children and 98 blood cultures from adults (53\%). Other organisms were isolated from six adults (3\%). An artificial airway was established in 352 children (73\%) and in 68 adults (19\%); the remainder were treated conservatively. Six children and two adults died.

Sweden has a high incidence of acute epiglottitis in children and the disease also occurs in adults. The importance of $H$ influenzae in the aetiology of epiglottitis in all age groups is confirmed, but in adults many cases occur without septicaemia. The mortality is currently very low.
\end{abstract}

Meningitis and epiglottitis are the most common and serious manifestations of invasive Haemophilus influenzae type $\mathrm{b}$ infections. The incidence of $H$ influenzae meningitis in children has been thoroughly studied in the United States and some western European countries. The age specific incidence in the most susceptible age group $0-4$ years has been reported to vary between 11 and 70/100 000/year. ${ }^{1-9}$ The variations can partly be explained by racial and socioeconomic factors. Certain high risk populations, North American Indians and Alaskan Eskimos, with incidence rates of 136 to $409 / 100000 /$ year have also been identified. ${ }^{10-12}$

Almost no data are available on the incidence of acute epiglottitis in children. An estimate of its importance can, however, be made from studies of invasive $H$ influenzae infections, in which the number of epiglottitis cases can be compared with the number of $H$ influenzae meningitis cases. According to all these studies meningitis is more common than epiglottitis. In two studies including all cases of epiglottitis based on clinical diagnosis, the epiglottitis cases numbered 62 and $65 \%$ of the $H$ influenzae meningitis cases, respectively. ${ }^{13} 14$ In other studies epiglottitis cases have been included only if the aetiology has been verified by isolation of $H$ influenzae from the blood, and it has not been stated how often blood cultures are obtained from children with epiglottitis. In such studies the numbers of epiglottitis cases have been 3 to $38 \%$ of the numbers of $H$ influenzae meningitis cases. ${ }^{5}$ 15-17

In contrast to all these studies, the incidence of epiglottitis in children was higher than the incidence of $H$ influenzae meningitis in the Göteborg area in Sweden during 1971-80. ${ }^{18}$ In this population based study, including all cases of epiglottitis based on strict clinical criteria in the age group $0-15$ years, the ratio between the epiglottitis and $H$ influenzae meningitis cases was $1 \cdot 44: 1$.

The aim of the present study was to calculate the incidence of acute epiglottitis in children in all Sweden in 1981-3 (population 8.3 million) and to compare the results with a previous study of $H$ influenzae meningitis during the same period, ${ }^{9}$ to see if the unique relation between the two $H$ influenzae manifestations reported from Göteborg ${ }^{18}$ is common to the whole country. Further aims of the study were to evaluate the importance of acute epiglottitis in adults and to study the mortality of the disease.

\section{Patients and methods}

The study is based on retrospective analyses of case records from patients admitted to hospitals in Sweden between 1 January 1981 and 31 December 1983 with a diagnosis at discharge of acute epiglottitis. Letters were sent to all departments of otorhinolaryngology, paediatrics, and infectious diseases and to certain intensive care units at small rural hospitals with a request that all patients with a discharge diagnosis of acute epiglottitis during 1981-3 and January 1984 should be identified and copies of the case records sent to us. A request was also sent to all departments of medicolegal medicine, where necropsies are performed on individuals dying outside hospital. From all 132 departments the requested information was made available.

Patients were included in the study if it was clearly documented that they fulfilled all of the following criteria: (a) a red and swollen epiglottis visualised at indirect laryngoscopy; (b) inspiratory stridor or difficulties in swallowing their own saliva or water; and (c) temperature $\geqslant 38^{\circ} \mathrm{C}$. Patients with concurrent tonsillitis, peritonsillitis, and certain other conditions were not included.

After exclusion of six non-Swedish residents and five patients whose case records could not 
be found, a total of 1101 patients with a date of admission during the three year period and a discharge diagnosis of acute epiglottitis were identified. Of these, 197 adults and 63 children were excluded as they did not fulfil the inclusion criteria. The most common reasons for exclusion were insufficient documentation of difficulties in breathing or swallowing $(n=70)$, concurrent tonsillitis or peritonsillitis $(n=85)$, normal temperature $(n=50)$, temperature not recorded $(n=11)$, or epiglottis not visualised $(n=10)$.

Population data from Sweden and its 24 counties for 1981-3 were obtained from official statistics. ${ }^{19}$

\section{Results}

CHILDREN $\leqslant 14$ YEARS

Of the $\mathbf{4 8 5}$ children fulfilling the criteria for epiglottitis, 286 (59\%) were boys and 199 (41\%) girls. The age distribution is shown in the figure. The mean age was 3.7 years; 39 children were younger than 18 months and the three youngest were 3 to 5 months old.

Blood cultures were obtained from 290 children $(60 \%)$ evenly distributed in different age groups (figure). $H$ influenzae was isolated from 267 of them (92\%) and 23 cultures (8\%) were negative. One hundred and thirty nine of the isolates were serotyped; all were type $b$.

The age specific incidence of epiglottitis in five year groups is shown in the table. There were no significant differences in the incidence between the 24 counties nor between south and north Sweden nor between counties with mainly urban and rural population.

In three counties (population age 0-14 years

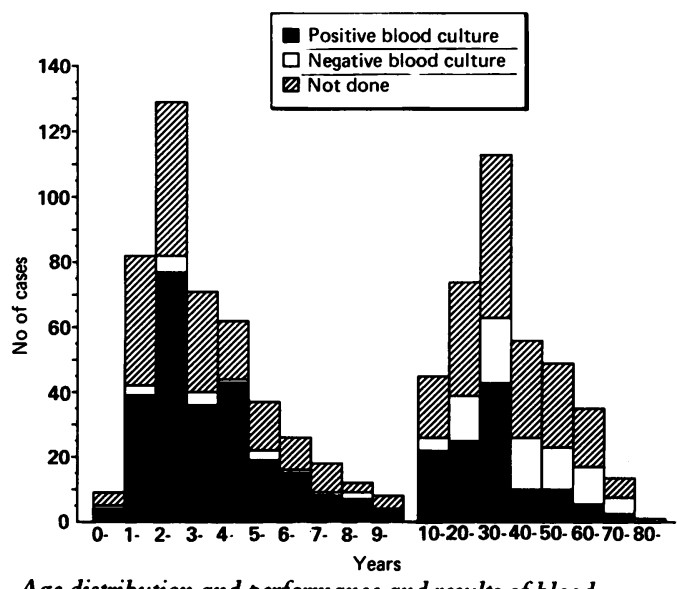

Age distribution and performance and results of blood cultures in 485 children and 356 adults with acute epiglottitis.

Age specific incidence (cases/100 000/year) of acute epiglottitis in Sweden 1981-3

\begin{tabular}{cccc}
\hline $\begin{array}{l}\text { Age } \\
\text { (years) }\end{array}$ & $\begin{array}{l}\text { Mean } \\
\text { population }\end{array}$ & $\begin{array}{l}\text { No } \\
\text { of cases }\end{array}$ & $\begin{array}{l}\text { Age specific } \\
\text { incidence }\end{array}$ \\
\hline $0-4$ & 477461 & 353 & $24 \cdot 6$ \\
$5-9$ & 530926 & 101 & 6.3 \\
$10-14$ & 563927 & 31 & $1 \cdot 8$ \\
$0-14$ & 1572314 & 485 & $10 \cdot 3$ \\
$\geqslant 15$ & 6752610 & 356 & $1 \cdot 8$ \\
All ages & 8324924 & 841 & 3.4 \\
\hline
\end{tabular}

148 094) blood cultures had been obtained from as many as 52 of 54 children and yielded $H$ influenzae in 49 cases. The incidence in these three counties was $12 \cdot 2$ if all cases were included and 11.0 if only cases verified by culture were included.

The cases were evenly distributed between the three years studied and there was no noticeable seasonal variation. Eleven children had concurrent conditions: neurological disorders $(n=3)$, Down's syndrome $(n=2)$, congenital heart disease $(n=2)$, diabetes mellitus, coeliaci, asthma bronchiale, and leukaemia. Twenty five children had other concurrent manifestations of $H$ influenzae infections: pneumonia $(n=18)$, otitis or sinusitits, or both $(n=6)$, and arthritis $(n=1)$. All children had had symptoms for less than 24 hours before admittance to hospital.

The mean duration of hospitalisation was four days (range 1-15). An artificial airway by intubation or tracheotomy was established in 352 of the children $(73 \%)$ while 129 were treated conservatively. Children treated conservatively were evenly distributed in all age groups. Blood cultures were obtained from 68 children treated conservatively and 57 yielded $H$ influenzae.

Six children died. Four of them died at home or were dead on arrival at hospital. The other two arrived at hospital deeply comatose with respiratory arrest and convulsions, respectively.

\section{ADULTS $\geqslant 15$ YEARS}

Of the 356 adults, 192 (54\%) were men and 164 $(46 \%)$ women. The age distribution is shown in the figure. The incidence in adults was $1 \cdot 8 / 100000 /$ year (table). The incidence did not vary significantly between the 24 counties.

Blood cultures were obtained from $185(52 \%)$ of the adult patients. $H$ influenzae was isolated from 98 of them (53 type b, 45 serotype unknown), from four Streptococcus pneumoniae, and from two Bacteroides spp. Blood cultures from 81 patients $(44 \%)$ were negative. As shown in the figure routines for obtaining blood cultures and isolation of organisms were not dependent on the age of the patient.

The adult cases were evenly distributed over the three years of study and there was no seasonal variation. Forty adults had concurrent diseases-for example, diabetes mellitus, cardiovascular and malignant diseases, chronic bronchitis, and chronic alcoholism. Four women were pregnant or had been delivered within hours before onset of symptoms. Fifty one adults had had symptoms for two to five days before they sought medical attention; the others presented within 24 hours. Twelve adults concurrently had other manifestations of $H$ influenzae infections: pneumonia $(\mathrm{n}=9)$, sinusitis $(n=2)$, and meningitis $(n=1)$.

Only 68 adults $(19 \%)$ required an artificial airway; the remainder were treated conservatively. Blood cultures were obtained from 142 of the 288 patients treated conservatively; 72 cultures were positive. Mean time of hospitalisation was five days (range 1 to 21 days). Two adults died. 


\section{Discussion}

Even though there are virtually no studies available on the incidence of acute epiglottitis in children, several studies from North America, Europe, and Australia have shown that this condition is less common than $H$ influenzae meningitis. Population based studies from England and Australia including all clinical cases have shown the ratio between epiglottitis and $H$ influenzae meningitis to be 62 to $65 \% .^{13}{ }^{14}$ Several studies including only epiglottitis cases with positive blood cultures have shown lower ratios. ${ }^{5}$ 15-17 In Alaskan Eskimos and Navajo Indians the incidence of $H$ influenzae meningitis is extremely high: $153-409 / 100000 /$ year in the age group $0-4$ years, but acute epiglottitis is rare or non-existent. ${ }^{1012}$ Virtually all cases of $H$ influenzae meningitis in these two populations occur in children younger than 18 months. Eskimo children above this age have high levels of anticapsular serum antibodies, ${ }^{10}$ and it is therefore not surprising that epiglottitis is rare among them as epiglottitis usually occurs after this age as shown in the present study and previously. ${ }^{13-18}$

In contrast to all these studies, the present study identified more children with acute epiglottitis (485) than with $H$ influenzae meningitis (470) in Sweden in 1981-3.9 ${ }^{9}$ The true difference between epiglottitis and meningitis cases is probably even larger for the following reasons. (1) In the meningitis study cases were identified both from files of hospital discharge diagnosis and laboratory records of cerebrospinal fluid cultures. Three per cent of all cases were found only from the second source. Epiglottitis cases could only be identified from one source, the hospital discharge diagnosis. (2) The criteria for inclusion of cases in the epiglottitis study were very strict. Several of the patients excluded may have had epiglottitis, but were excluded because of insufficient documentation or less pronounced symptoms. (3) Some case records from children with epiglottitis were not found while all records from meningitis patients were found. Thus the study confirms the previous study from Göteborg, Sweden, which also showed a higher incidence of epiglottitis than $H$ influenzae meningitis in children. ${ }^{18}$

As a possible reason for the high incidence of epiglottitis in Swedish children we suggest that the socioeconomic situation with small families, no overcrowding, and little use of day care centres before the age of 18-24 months, may be important. This may lead to less chance of exposure to $H$ influenzae type b during the first years of life when invasive $H$ influenzae infections are more likely to manifest as meningitis than as epiglottitis. It may also lead to a fairly large proportion of non-immune children after the age of 18-24 months when the disease is more likely to manifest as epiglottitis.

In view of the development of vaccines against invasive $H$ influenzae type $b$ infections it is important to evaluate the impact of invasive $H$ influenzae infections on morbidity and mortality in children. The combined incidence of $H$ influenzae meningitis ${ }^{9}$ and epiglottitis (present study) in Sweden in the whole child population 0-14 years was 20/100 000/year. The risk for an individual child to develop either of these two disease manifestations before the age of 15 years can be estimated to one in 330 .

$H$ influenzae type $\mathrm{b}$ infections have been considered to occur almost only in children, but during the last three decades several reports have appeared describing acute epiglottitis and other invasive $H$ influenzae type $\mathrm{b}$ infections in adults. ${ }^{20-26}$ To our knowledge only one population based study (from Rhode Island) of the incidence of acute epiglottitis in adults has been performed. ${ }^{26}$ In this study the incidence was $0 \cdot 97 / 100000 /$ year. In the present study a higher incidence in adults, $1 \cdot 8 / 100$ 000/year, was found, even though we applied more rigid criteria for inclusion, for example, a temperature $\geqslant 38^{\circ} \mathrm{C}$. During the same time period the incidence of $H$ influenzae meningitis in adults in Sweden was only $0 \cdot 15 / 100000 /$ year. $^{9}$

The present and previous studies show that $H$ influenzae is also the most common cause of acute epiglottitis in adults. ${ }^{23-26}$ In our study $53 \%$ of all blood cultures obtained from adults yielded this organism. Other organisms, notably pneumococci, can, however, also cause epiglottitis in adults. ${ }^{25} 27$ The clinical presentation was less dramatic in adults than in children. Artificial airway was established in only $19 \%$ of adults compared with $73 \%$ of the children, and $14 \%$ of the adults came to hospital more than 24 hours after onset of symptoms.

In conclusion, this study has shown a remarkably high incidence of acute epiglottitis in both children and adults in Sweden. The importance of $H$ influenzae in the aetiology of the condition was confirmed in all age groups, but many adult patients have negative blood cultures. The case fatality rate is very low in all age groups.

We thank all doctors and secretaries at hospitals in Sweden who helped identify cases and made their data available to us. The study was supported by the First of May Flower Annual Campaign for Children's Health, the Foundation 'Samariten', and the Faculty of Medicine, University of Göteborg.

1 Smith Jr EWP, Haynes RE. Changing incidence of Haemophilus influenzae meningitis. Pediatrics 1972;50:723-7.

Tarr PI, Peter G. Demographic factors in the epidemiology of Haemophilus influenzae meningitis in young children. Haemophilus influenzae

3 Santosham M, Kallman CH, Neff JM, Moxon ER. Absence of increasing incidence of meningitis caused by Haemoof increasing incidence of meningitis caused by Haemophilus influenzae type b. F Infect Dis 1979;140:1009-12.
Parke JC Jr. Capsular polysaccharide of Haemophilus influenzae type by as a vaccine. Pediatr Infect Dis 1987; 6 : 795-8.

5 Murphy TV, Osterholm MT, Pierson LM, et al. Prospective surveillance of Haemophilus influenzae type $b$ disease in Dallas County, Texas, and in Minnesota. Pediatrics 1987; 79:173-80.

6 Goldacre MJ. Acute bacterial meningitis in childhood. Incidence and mortality in a defined population. Lancel 1976;i:28-31.

7 Claesson B, Trollfors B, Jodal U, Rosenhall U. Incidence and prognosis of Haemophilus influenzae meningitis in children in a Swedish region. Pediatr Infect Dis 1984;3:35-9.

8 Spanjaard L, Bol P, Ekker W, Zanen HC. The incidence of bacterial meningitis in the Netherlands-a comparison of three registration systems, 1977-1982. F Infect 1985;11: three reg8.

9 Trollfors B, Claesson BA, Strangert K, Taranger J. Haemophilus influenzae meningitis in Sweden 1981-1983. Arch

10 Ward JI, Margolis HS, Lum MKW, et al. Haemophilus influenzae disease in Alaskan Eskimos: characteristics of a influenzae disease in Alaskan Eskimos: characteristics of a
population with an unusual incidence of invasive disease. population with an un
Lancet $1981 ; \mathrm{i}: 1281-5$

11 Yost GC, Kaplan AM, Bustamante R, et al. Bacterial meningitis in Arizona American Indian children. Am $\mathcal{F}$ Dis Child 1986;140:943-6. 
12 Coulehan JL, Michaels RH, Hallowell C, et al. Epidemiology of Haemophilus influenzae type $b$ disease among Navajo Indians. Public Health Rep 1984;99:404-9.

13 Broughton SJ, Warren RE. A review of Haemophilus influenzae infections in Cambridge 1975-1981. F Infect 1984;9:30-42.

14 Koo W, Oley C, Munro R, Tomlinson P. Systemic Haemophilus influenzae infection in childhood. Med $\mathcal{J}$ Aust 1982;2:77-80.

15 Granoff DM, Basden M. Haemophilus influenzae infections in Fresno County, California: a prospective study of the effects of age, race, and contact with a case on incidence of effects of age, race, and contact with a
disease. F Infect Dis 1980;141:40-6.

16 Michaels RH, Schultz WF. The frequency of Haemophilus influenzae infections: an analysis of racial and environinfluenzae infections: an analysis of racial and environ-
mental factors. In: Sell SHW, Karzon DT, eds. Haemophilus influenzae. Nashville: Vanderbilt University Press,

17 Peltola H, Virtanen M. Systemic Haemophilus influenzae infection in Finland. Clin Pediatr (Phila) 1984;23:275-80.

8 Claesson B, Trollfors B, Ekström-Jodal B, et al. Incidence and prognosis of acute epiglottitis in children in a Swedish region. Pediatr Infect Dis 1984;3:534-8.

19 National Central Bureau of Statistics. Official statistics of Sweden. Population part 3. Stockholm: National Bureau of Statistics, 1980-1983.
20 van Alphen L, van Dam A, Bol P, et al. Types and subtypes of 73 strains of Haemophilus influenzae isolated from patients more than 6 years of age with meningitis in the Netherlands. f Infect 1987;15:95-101.

21 Spagnuolo PJ, Ellner JJ, Lerner PI, et al. Haemophilus influenzae meningitis: the spectrum of disease in adults. Medicine 1982;61:74-85.

22 Quintiliani R, Hymans PJ. The association of bacteremic Haemophilus influenzae pneumonia in adults with typable Haemophilus influenzae pneumon
strains. Am $\mathcal{I}$ Med 1971;50:781-6.

23 Trollfors B, Claesson B, Lagergård T, Sandberg T. Incidence, predisposing factors and manifestations of invasive Haemophilus influenzae infections in adults. Eur $\mathcal{f}$ Clin Microbiol 1984;3:180-4.

24 Johnstone JM, Lawy HS. Acute epiglottitis in adults due to infection with Haemophilus influenzae type $b$. Lancet 1967;ii:134-6.

25 Gorfinkel HJ, Brown R, Kabins SA. Acute infectious epiglottitis in adults. Ann Intern Med 1969;70:289-94

26 MayoSmith MF, Hirsch PJ, Wodzinski SF, Schiffman FJ. Acute epiglottitis in adults. An eight-year experience in the state of Rhode Island. $N$ Engl F Med 1986;314:1133-9.

27 Branefors-Helander P, Jeppsson PH. Acute epiglottitis. A clinical, bacteriological and serological study. Scand $\mathcal{J}$ Infect Dis 1975;7:103-11. 